\title{
FROM THE BIT TO \\ THE PIT: POETICS \\ OF THE FINANCIAL \\ MARKET
}

ELIE AYACHE

ELIE AYACHE was born in Lebanon in 1966. Trained as an engineer at L'Ecole Polytechnique of Paris, he pursued a career of option market-maker on the floor of MATIF (I987-I990) and LIFFE (1990-1995). He then turned to the philosophy of probability (DEA at la Sorbonne) and to the technology of derivative pricing, and co-founded ITO 33 , a financial software company, in 1999. Today, ITO 33 is the leading specialist in the pricing of convertible bonds, in the equity-to-credit problem, and more generally in the calibration and recalibration of volatility surfaces. Ayache has published numerous articles on the philosophy of contingent claims. He is the author of The Blank Swan: The End of Probability (2010), and of The Medium of Contingency: An Inverse View of the Market (2015). 
When pondering the question of present-day communication and whether the deluge of data might end up obscuring information, it is difficult to content oneself with part of the universe and not to consider the total communication system that the whole universe is. Philosophers of quantum computation like Seth Lloyd equate the universe with a gigantic quantum computer, in which scripts keep being generated at random, eventually constituting stable physical systems and autonomous life forms. ${ }^{\mathrm{I}}$ Markus Gabriel teaches us, on the other hand, that the world is a bigger entity than the universe. The universe is the collection of all physical systems produced by quantum code, with a perfect duality between object and property, physical system and physical law, whereas the world is the collection of all things imaginable. There is the physical world or universe, of course; but there is, additionally, the world of dreams, the world of literature, the world of man, etc., and perhaps even the world of communication.

The universe exists, while the world doesn't, according to Gabriel. $^{2}$ The collection of all things cannot exist. Speaking of the world is usually the indication of a shift in discourse, from physical to metaphysical. One typically speaks of the end of the world, not of the end of the universe, and in the typical limiting movement that worries us, in which the excess of information becomes equivalent to its lack, or density becomes equivalent to the void, or total memory becomes equivalent to a blank; it is the world that becomes implicitly our object of discourse - a metaphysical system rather than a physical one.

We claim that the financial market is of the same category as the world and is also a metaphysical system, not a physical one. Probably for this reason, it gets no mention in the computational renderings of the universe, such as Seth Lloyd's. Yet we wonder: Can't the market be considered a consequence of the randomness that is produced by the universe, similarly to physical systems? The market exists, after all. Can't it also be considered a script, perhaps even an unavoidable one, obtained by the computational universe? Or does the world of man have necessarily to get involved in its making? Should books and poems be considered the result of the computational universe too?

Perhaps the necessity of emergence of the market within the computational universe is due to the possibility of annihilation of any script and of the failure of communication - a possibility that necessarily exists. Indeed, the condition of possibility of communication, or its material medium, is at the same time the condition of its impossibility, in case this medium is annihilated. And it suddenly strikes us that the entire analysis of the universe as a prodigious quantum computer might

I Seth Lloyd, Programming the Universe: A Quantum Computer Scientist Takes on the Cosmos (New York: Alfred A. Knopf, a division of Random House, 2006).

2 Markus Gabriel, Why the World Doesn't Exist (Cambridge and Walden: Polity Press, 2015). 
be based on a notion of randomness and of its communication channel that ignores writing and its (indispensable) other face, which is the necessary possibility of annihilation of the written record. ${ }^{3}$

Thus, it ignores the exchange of written records, which is the other face of their material nature. To anticipate our discourse of the market, the reason financial contracts are materially exchanged between individuals, and priced solely in this fashion instead of being valued by an algorithm, is that they can be annihilated or defaulted. The market exists and is not programmable, the market is not redundant, because of the necessary intervention of the world (and of the end of the world) in the universe.

The computational vision of the universe can only comprehend the financial market as one among other data-producing systems. Nobody sees the market as a means of communication with something totally different, with the end of the communication, for instance. This is because nobody wants to antagonize the very archaeology of thought that produces the computational vision; nobody wants a criticism of the notion of state (computational or otherwise).

Can it reasonably be argued that the financial market presents itself in the universe because of the forgotten criticism and that if one were to supplement an analysis like Seth Lloyd's, which is great for saying what the universe is in effect, with the clause of failure of communication, or with the channel that communicates with the event and with the end of the world, then this revolutionary medium which communicates with the incommunicable would be the market (or its generalized notion)? We fear this new category might elude any positive analysis. For, instead of speaking of information and of communicational content, we speak of systems that lie in no computational state - of systems that lie in a total absence of state, which is a state of total preparation for writing and for communication with the event.

What good would writing be, indeed, if not to communicate with the event? What good would thinking be-all the invisible things that one keeps pushing in a corner of the world - if it were all reducible to code, and positively transmissible by the channel of communication?

\section{ABYSS OF THE CONCRETE WORLD}

The bit, or the basic unit of information, is a random variable $\mathrm{X}$ that switches between o and I. It is communicable because the variable $\mathrm{X}$

3 Not to mention the converse situation, in which the written record survives the death of the two communicating parties and literally breaks free from the initial context of communication. This is not an accident, according to Derrida, but the very definition of writing, which therefore can never be reduced to a means of communication. On the contrary, it signals the failure of communication qua transmission of meaning or intention. See Jacques Derrida, 'Signature, Event, Context', in Margins of Philosophy, trans. by A. Bass (Chicago: Chicago University Press, 1985). 
is real (in a sense we shall define shortly) and measurable. It is the answer to a question; it is an output. As a matter of fact, it is a function of something very strange that abstract probability theory recognizes as the 'sample $\omega$ '.

Indeed, the random variable $\mathrm{X}$ is a mapping between an abstract space, the 'universe' or 'sample space W' which is meant to remain unanalyzed, and a set which will be subject to mathematical analysis, typically the set of real numbers:

$$
\begin{aligned}
\Omega & \rightarrow \mathbb{R} \\
\omega & \mapsto X(\omega)
\end{aligned}
$$

Ironically, the sample $\omega$ is the index of the concrete situation or concrete case, of that which has concretely to happen in the world-call it the random draw - in order that the random variable may admit a value. The sample $\omega$ isn't a variable; it is something else. A 'variable' represents the lowest level in mathematical analysis; and rightly so, probability theory reserves that term to $\mathrm{X}$ and not to $\omega$.

The Markov system is the paradigm of communication systems and the important notion of state of that system is in truth derived and not primitive. It is the consequence of two characteristic properties of Markov chains: first, that singletons of values of the random variable are measurable and can enter in the definition of conditional probability; second, that the past and the future are independent, conditionally on the present state that has just been defined. This enables the definition of the probability of transition between states, which is therefore a derived notion too. Communication, state and transition between states are thus indissolubly linked, but they all derive, as we will see, from something infrastructural and more fundamental-literally, the incommunicable.

Indeed, the basic unit of information, o or $\mathrm{I}$, or the bit, is not the deepest level of information. It doesn't get to the bottom of things. It is not the 'absolute' information because it is the state of a random variable, therefore the reflection of a point of view. It presupposes a context or a preliminary choice of the algebra of events (or possibilities), which is always relative. One has to edit the absolute concrete in order to produce the real. Information is real; it is definitional of the level of reality, in our construction, because it is the answer to a well-posed question. As real, however, information is underlain by the concrete sample $\omega$, by that which has absolutely concretely happened in order to make the value o or I appear.

Let us add, parenthetically, that probability should be the first arithmetic and the first science of numbers, the one tying them up with the concrete world and with 'what has concretely happened'. Nothing happens in analysis, by contrast. Thus, the truly fundamental 'variable' should be the one that indexes the concrete world and is rooted in it; it should be the concrete sample $\omega$, which is not a variable in the sense of 
analysis. In any deconstruction enterprise, one should first tackle this infrastructure. The conditions of impossibility of communication are part of the concrete world that is presupposed by the screen of reality and by the editing of the real, that is to say, by abstraction.

Yet, the sample $\omega$ cannot be measured. We cannot measure what has absolutely concretely happened. There is no communicable information contained in this, no question that would map the states of a random variable and would admit of an answer. This literally communicates with the incommunicable, with the abyss of information that matter constitutes in any case.

Why this word, matter? Why is matter an abyss of information? Because the event that would bring communication and the whole world to an end belongs to no previously established algebra of events. It escapes any context, any frame of reference, and for this reason it is absolute like matter. It "carries with it a force of breaking with its context' ${ }^{4}$ On the other hand, $\omega$, or the concrete sample of abstract probability theory, is the first sign of irruption of matter into the formalism, or of thought thinking matter, and for this reason it is abysmal.

One should not confuse mass and matter. The semblance of infinity that contemporary mass media produce should not be equated with the infinity of the abyss in which the random sample $\omega$ is thrown. Indeed, we deal with intensity, in the latter case, not with extensity and quantity; we deal with the unmeasurable mass of that which has absolutely concretely happened.

The concrete world is not just an accessory, in abstract probability theory. Our first impression is that the concrete detail is supplemental and concerns only the contingent world. However, detailing the colour of the dice or the colour of the sky above it is only an image and an illustration. What really counts in the concrete world is that the descent in its abyss should be indefinite, that is to say, infinite. That 'it' happens, without further specification, is in itself an abysmal notion. The logic of randomness claims this infinity, because it claims repetition. If it were not for the concrete world and for the indefinite level at which the line of abstraction is drawn (which just underlines the infinite play between thought and matter), there wouldn't be the notion of independence and repetition, which is fundamental for randomness.

\section{RANDOMNESS AND THE EVENT}

Anyone who throws the dice once throws it an infinity of times. This is because the dice is there, concretely. This intensive infinity is only the

4 Jacques Derrida, 'Signature, Event, Context', in Margins of Philosophy, Alan Bass trans. (Chicago: Chicago University Press, 1985). 
expression of the stroke of the matter of the dice, as it gets inscribed in thought. The strong law of large numbers, or the convergence of the frequency of appearance of a particular face when throwing the dice until the end, is only the indication of localized matter. Given the bottomless pit and the bottomless question of what has absolutely concretely happened, given that everything that will have ever happened in the history of the universe could be virtually recounted as a single sample that the world has drawn from the urn, once and for all, this fall is in reality always suspended, the line of abstraction is always drawn at some level of the concrete abyss, and a point of view is always adopted.

A local physical object is thus carved out of the world, and this automatically entails that the object has multiple faces and that multiple samples can produce the same face. This brings about repetition, therefore randomness in the ordinary sense and convergence of the frequency. Now that we have suspended the fall and broken the 'once and for all', we can only repeat. For otherwise, we are never done with the first sample, never done answering the question of what has absolutely concretely happened.

Ultimately, the traditional logic of randomness exhausts history by the notion of density and totality, by a sample space that is supposed to contain all that has ever been the case from the beginning until the end of times. In its ontology, the event of a meteorite falling and killing the players who are throwing the dice is not a radically emergent event. Surely enough, it wasn't imaginable or perhaps even conceivable beforehand; it wasn't part of the game of dice and its algebra of measurable events; it belonged completely outside the context of the game. However, according to the totalizing logic of sample space, the case that brought it about has always been part of the big urn that the world is drawing its samples from.

The event becomes conceivable after it appears. Just as the face of the die never happened as such, but something concrete happened instead, the sample $\omega$, and made the face of the die appear, just so, something happens now, making the meteorite appear, yet has always been an element of sample space. Now that the falling meteorite has become a conceivable event, the algebra of events is recalibrated and further refined (we advance the filtration by one step) and a new 'face' is added to the die. It is as if we were suddenly able to look more closely into the detail of the concrete situation and the meteorite was only a previously indiscernible detail.

Another name for this twisted metaphysics is the backward narrative. The backward narrative is an attempt to bring history to an end, as it implies that the end has always been at the beginning. A liberating alternative would be to 'stop' history by the void and not by density, or equivalently by the absolute capacity, by claiming that what has 'absolutely concretely happened' is that everything, anything, can happen. 
It is characteristic of communicable information (of its algebra, of its filtration) that one be able to regress in the abyss, at each iteration, and say: 'This is the way things must have happened from the beginning; this is what my algebra of events must have always been, from the beginning.' An alternative way of placing oneself at once in the infinity of 'what has already happened,' a progressive and not a regressive way, consists in saying that it hasn't yet happened. Intensity, not extensity. The infinite intensity is such, in this case, that one writes effectively after the event, before it. Although the event doesn't exist yet, although it is absolute difference (all the more absolute as we don't know what the event is different from), we have to establish contact with it and communicate with it, in a single stroke.

To this end, we must consider a transformation of probability that tests the limits of the logic of randomness and takes up the total set of cases, or universe $\mathrm{W}$, to transform it into a world. This transformation will produce the financial market and will throw the abyss forward. We won't be reconstructing the universe, anymore, in an ever-regressive fashion, but finally meeting with the world. This amounts to saying that the reality of the market will replace the 'reality' of abstract probability theory (its algebra of events) and will be, from now on, in contact and in continuity with the absolute concrete. We won't be tumbling down the abyss of the absolute concrete anymore, in search for its absolute detail, because it will be projected before us at once, for all times.

\section{MONEY}

Communication by the market, as medium of contingency, is communication with the infinity of the concrete situation, an intensive and not extensive infinity. It is not a set whose elements can increase or decrease in number, and not even a variable that can run over a total.

The financial market is usually confused with the deluge of statistical data. This is because the argument from the derivatives has been overlooked, a qualitative and not a quantitative argument, as we shall see, which issues from the operation of conceptualization and not of abstraction. Indeed, we shall interpret the successive writing of derivatives and their successive re-immersion in the trading pit as a succession of degrees of conceptualization that will never rise above the immanence of the exchange and will always reaffirm the trading pit as only concrete, not as successive levels of abstraction of that concrete. The distinction we make between conceptualization and abstraction is thus fundamental. But first, a word about money.

The argument against the state and possibility, or against communication and probabilistic transitions, should stem from the local and not the global, from what's hidden under our very feet. Jean-Yves Girard, whose Le Fantôme de la transparence (or ghost of transparency) was 
certainly an inspiration to the title of the present volume, is right to speak of the implicit, in the particular instance, and particularly so of money. ${ }^{5}$ Money is implicit, he writes, because "it represents everything, or rather, anything." From which I speculate that it corresponds to the intensity of the abyss; that it 'communicates' with the incommunicable and not with the extensive collection of data or the extensity of information. The concrete sample $\omega$ was invariably implicit too, under the surface of measurable things. "Thought belongs to the domain of the implicit," writes Girard, "while the explicit limits itself to verification." So the fascinating question becomes: What would be the alternative, to both the world and communication if the abyss of money became the only concrete?

The alternative to the abstraction of probability theory is the conceptualization of the trading pit. This is our thesis. There is infinity in money because money is at the same time its own ground and own abyss. It lends itself to speculative bubbles and to market crashes at the same time, and not in alternation. The pit presents us with the vision of its interior because of this spontaneous infinity. This vision from the inside (consisting in riding the channel of money instead of measuring values and verifying information from the outside) is the one, in our view, that requires conceptualization instead of abstraction.

At play is the implicit character of money. When money, which applies to anything, is applied to itself, it becomes the only end of the world, and the trading pit closes itself on us. We no longer buy the stock to acquire a value (that of the issuing company) but only to purchase the future price movement of the trading stock. We then can only make money or lose money. The stock price can only go up or go down. There are no other measurable events in this world. And this is not an abstraction over a concrete sample that might be infinitely richer in the point of unmeasurable detail. This is simply money.

People buy the stock because its price could go up, and its price goes up because people buy it. Or they sell it because its price could go down, and its price goes down because people sell it. Money is in itself the primary abstraction and then it becomes the only concrete. It screens off any other concrete. There Aren't even two events in this world (the price going up or down). There is only money, which becomes more fundamental than randomness and perhaps even time.

As soon as the stock exchange (or the trading pit) is given, randomness is given. The infrastructure of sample space is no longer needed. Once the free exchange is accepted, speculation, or the market looking at itself reflexively, becomes the only ground, a constantly moving ground.

5 Jean-Yves Girard, Le Fantôme de la transparence (Paris: Éditions Allia, 2016). All quotes are my translation. 
The movement of the stock price becomes the only commodity and precisely derivatives (a.k.a. contingent claims) are written, which pay off if certain price paths are realized. And now the implicit character of money plays the crucial role. It is such that the payoff those derivatives return when they expire is made of the same money as the one that is made or lost in the process of trading their underlying stock. This, after the Nobel-prize winning discovery by Black, Scholes and Merton (BSM) in $1973,{ }^{6}$ allows derivatives to be replicated by a self-financing dynamic trading strategy involving the underlying stock, and hence it allows them to be re-immersed, in their turn, in the same market and in the same trading pit. This invariable reversion of the overlying to the underlying, of the derivative (no matter how complex) to the basic trading floor, is the implicit definition of the market, in our view. ${ }^{7}$ The money in which one would be tempted to value the derivatives externally as lotteries is, once again, of the same nature as the one in which they are traded and admit a price.

Money doesn't have two faces, $o$ and I. Money is not a coin we flip or a die we cast. Money is the concrete world become liquid. The entire abyss slips itself inside money and now constitutes a ground on which we stand in order to project the entire concrete world in the opposite direction. That money should have no other meaning but to be made or lost, that money itself could become worthless and should have the end of the world as its other face (that is to say, no other face at all); that this should be the implicit definition of money is what enables us to replace the explicit state, o or $\mathrm{I}$, by the single self-financing trading strategy and by the single face of money.

\section{THE TRADING PIT}

Trading is not a matter of drawing a sample from the urn. Trading means randomness. By the efficient market hypothesis, the stock price cannot but be volatile. If asked what the meaning of the market is, what the concept of the market is, we should answer: volatility. We may not know what the value of anything is, because there is only price and price is volatile, but now we know volatility. Volatility becomes the value of the market. Thus, it is the very concept of the trading pit of the underlying stock (volatility), and not its abstraction, that BSM have transformed into the value of the derivative written on that underlying, a value soon to join the trading pit again and to become price again.

6 Fisher Black and Myron Scholes, The pricing of options and corporate liabilities, The Journal of Political Economy, 8 I(3), May-June 1973, 637-654.

7 Elie Ayache, The Medium of Contingency: An Inverse View of the Market (Basingstoke and New York: Palgrave Macmillan, 2015). 
What is there to abstract, anyway? Remember that money and trading are the only concrete, and that there is no deeper, underlying concrete. Remember that the trading pit is the only world. So it cannot be abstraction that we are achieving here, no longer a cut, but a conceptualization of the whole thing. This is an alternative schema of thought altogether. We are thrown in the abyss of trading from the beginning and BSM show us, via Brownian volatility and the self-financing dynamic trading strategy, that the contingent payoff is the result of conceptualization of the trading of the underlying stock, rather than its mere probabilistic abstraction as a random process. In the concept of trading a stock, there is both the randomness of its price and the variability of the size we are holding. This newly synthesized contingent payoff can then only become a materially written contingent claim and be traded in its turn. This is the crux of the matter.

When money and trading are the only ground, a thing can be conceived, that is to say, carved out of the concrete world, only if it is exchanged. This is the different order of time and matter we are dealing with. There is always infinity in the way thought joins itself with matter then separates itself from it. One infinity results in the usual view of randomness and of the concrete situation, and another results in the exchange. One is backward, the other is forward.

One really has to understand how different the two masses are from each other, and how exclusive of each other. Something in the archaeology of thought places the abyss of the concrete situation on one side, and the same thing makes it so that one possible transmutation of the mass is the abyss of the exchange. In one case, the realm of abstraction generates random variables and values of random variables, or the vision from outside. In the other case, the single face of money (identity between the ground and the abyss or between the speculative bubble and the market crash) generates contingent claims and prices, or the vision from inside in which the event is already an integral part.

By contrast with the infinite but regressive descent in the abyss of the concrete situation, writing, or the immersion in the trading pit, is infinite and progressive. To give oneself a total and pre-existing sample space, over which only to draw the line of abstraction, is what causes the finite character of this regressive infinity: an infinity that is not spontaneous. The culprit is thus the schema of abstraction, or the traditional logic of randomness. It causes the recalibration of the algebra of events always to take place as a backward narrative, while denying, every time, that it has gone backwards in this fashion. It causes thought constantly to be hiding from itself and not being in sync with itself.

Money goes forwards, by contrast. By the implicit definition of the market and of money, the prices of all derivatives are all given at once, despite the seemingly sequential order in which those derivatives are written and traded. As prices, they vary from any probabilistic 
valuation, before they even move. The recalibration process of the algebra of events, which was always stopped at a finite level and always restarted in total denial of the previous stage, is now spontaneously unfolded, going forward. Time is no longer absorbed by the abyss. It liberates itself into historical time.

There is no communicable information in the market, because any piece of information, any level of reality and of abstraction that one reaches in order to communicate is at once translated into the exchange. Any stage of abstraction that would allow an algorithm to create its reality and its referents can be concretely written as the payoff of a contingent claim, and the latter, precisely because of matter and of the first affliction which is, in the present instance, that anything written is traded at once, can only be united with the first (and only) level of reality, the reality of trading.

Strangely enough, this homogeneity (or hegemony) of price is the reason why some thinkers have lost any interest in the market and have considered it, from the beginning, as just a shapeless mass of transactions, or simply chaos. They did not perceive the richness in the back and forth movement of trading and writing. Trading a stock is the same as writing a derivative on that stock, is the same as trading the derivative, is the same as writing a derivative on the derivative, is the same as trading the second derivative in turn, etc. In a single stroke, the infinity of the abyss of the concrete world is handed back from the other end, never to be totalized again in a universe of cases.

This singularity of the market, which enables it to communicate directly with the singularity of the event, makes it foreign to any notion of progressive unveiling of information. For this reason, the alternative metaphor is, to my eyes, that of the blank that is filled by the single stroke of writing and by the single poem. The end of the world can only be communicated by writing. Under no circumstance does a poem or a book communicate information.

Thus style is the opposite of information. Books and poems communicate the former, not the latter. Another meaning of style is stylus, or the sharp and pointed instrument with which to impress matter and engrave it. One speaks of an amount of information or of a filtration indexed by time. Information is metrical - either volumetric, or chronologic - whereas the book is intense and possesses only writing style. It may have the volume of an entire world and may communicate with the end of the world; it nevertheless constitutes a single stroke. In Search of Lost Time is a single directed stroke; it is not a collection of words. For this reason, one cannot subtract a single word from it. 
